\title{
Le présent altéré
}

\author{
Gemma Serrano \\ (Facult de Théologie Notre-Dame, Collège des Bernardins, \\ Parisgemma.serrano@collegedesbernardins.fr)
}

« Le présent des choses passées, le présent des choses présentes, le présent des choses futures » est en train d'être modifié, telle est l'affirmation que nous défendons ici. Les conditions techniques et sociales actuelles interrogent les possibilités du rapport au passé et à l'avenir dans l'expérience du présent qui ne cesse de se transformer à mesure qu'évoluent les artefacts et les sociétés. Notre hypothèse de travail est que "le numérique ", ayant dépassé le statut de systèmes de support de la vie, est en train d'instaurer un nouvel affect temporel de l'existence. Notre propos consistera à décrire les changements du rapport du sujet au présent par l'accroissement de la " mémoire digitale », la progressive disparition de la remémoration et la disparition de l'attente.

\section{Le présent des choses présentes, passées et à venir}

Il importe de voir que la distinction entre la mémoire du sujet et celle de la mémoire numérique tend à s'amenuiser dans la mesure où, nous semble-t-il, la "face objectale" de la mémoire numérique s'identifie àla mémoire subjective. Le rapport des technologies numériques àla modalitétemporelle du futur se base essentiellement sur l'anticipation. Le fonctionnement des technologies numériques mises au service de l'industrie des données repose, entre autres caractéristiques, sur la possibilitéde prédire et de produire les comportements futurs des individus, sur la base de la collecte et du traitement algorithmique de leurs traces. Dans la mesure où les environnements numériques s'adaptent en « temps réel » aux profils des individus et présentent un possible qui peut correspondre aux traces laissées, la prédiction des actions futures se confond avec la reproduction des comportements passés. Cela est très clair, par exemple, dans la suggestion des achats.

Cette anticipation du futur nie la volonté et l'attente du présent. L'attente qui est le présent des choses à venir se situe en avant du présent. Un avant qui n'est pas le résultat causal et consécutif du présent. Autrement dit, le futur n'a pas à se confondre avec l'ordre de la prévision ou de la prédiction à partir du passé. Le futur à l'avant du présent exige, d'une certaine manière, de se comprendre comme le corps penchéen avant, dans l'imminence et l'urgence de déchiffrer ce qui est au-delà et veillant ce " plus », cet excès, ce qui est extrême et se tient àl'avant. On pourrait dire que le « présent des choses à 
venir » se tient dans l'attente du présent des choses présentes, et dans la tension qui désire ce qui est presque là ou pas du tout. Le futur exprime rupture, nouveauté, inattendu. Il est dans la présente tendance vers, aspiration, désir de l'avenir. Autrement dit, le présent couve le futur tant individuel que collectif.

Mais dans l'anticipation, l'expérience du présent des choses à venir est sécurisée par ce qui est prévisible, toute incertitude et rupture est éliminée. Le sujet s'attend à ce qu'il connaît déjà et est conforté par la prévision. Dans cette programmation du futur à travers des traces, c'est l'attente du présent qui semble être totalement annihilée.

Les traces du passé déterminent les choix futurs sans marge d'erreur ou de nouveauté, voire de rupture. Cette anticipation ne se situe pas au-delà du présent, veillant ce "plus », cet excès qui lui est propre comme présent. Non prae mais ante, c'est-à-dire devant ce qui a de la place capere. Le présent des choses à venir change ainsi de position, il se tient devant le passé, face aux capacités déjà exercées et n'a rien d'autre à faire que de s'occuper de prévoir. Il n'est plus à l'avant, en excès, mais rabougri sur le devant de ce qui a déjà été. Praesens signifiant "ce qui est à l'avant de moi », ce qui est presque là, sans délai, urgent, imminent, immédiatement actuel, présent. C'est le sens de la préposition latine prae qui rend clair le sens : non ce qui est là, « en face de », «devant», mais ce qui est au-delà, à l'avant, sans une continuité nécessaire avec ce qui est postérieur (Benveniste 1966, 132-139).

Un autre changement du présent concerne le présent des choses passées qu'est la mémoire. La traduction en données de la mémoire enlève progressivement la lutte contre l'oubli, la crainte d'avoir oublié et la recherche du souvenir. Nous sommes de moins en moins capables d'interpréter le monde grâce à notre mémoire. En revanche, nous sommes interprétés par l'intermédiaire des données que nous laissons et qui gardent mémoire de nous.

La « mémoire » est traduite automatiquement en données digitales. Mais cette traduction ne permet pas une interprétation de la part des individus car ils sont dépossédés de leurs traces, ils sont rendus incapables de partager, de se réapproprier, d'interpréter et de transformer leur mémoire et de l'oublier.

De même, les traces en nous que le présent des choses passées nous fait éprouver disparaissent aussi. L'écho du passé dans le présent devient muet et peu d'expériences du présent laissent une trace en nous et peuvent nous permettre de construire une narration à partir de nos vies individuelles. Si on prend à notre compte l'idée avancée il y a près d'un siècle par Walter Benjamin: «nous devenons de plus en plus riches d'épisodes d'expérience, mais de plus en plus pauvres en expériences vécues (Erfahrungen)». Autrement dit, nous devenons de plus en plus riches en mémoire numérique et de plus en plus pauvres en présent ; plus de «queue de comète » ou de « résonance » comme dirait Husserl. L'expérience vécue perd ses privilèges. 
L'expérience du présent des choses présentes, passées et futures, se transforme et devient muette, traduite en données, captive et automatisée. La mémoire autant que l'attente sont simultanées, leur négation dans un présent figé enlève la tension propre au présent et modifie l'affect.

\subsection{Le présent des choses passées est traduit en données}

Les moments vécus traduits en données perdent de leur épaisseur sensorielle, rendent impossible une nouvelle réécriture ou modification dans le moment présent et donnent congé à l'oubli. Ceci est démontré dans un épisode de la série télé britannique Black Mirror écrite par Charlie Brooker et Jesse Armstrong. Cet épisode met en scène la conservation sans limites de la mémoire du sujet et l'accès et la disponibilité de chaque seconde vécue. Le personnage, Liam (Toby Kebell) rentre d'un entretien d'embauche et regarde sa femme Fi (Jody Whittaker) discuter avec un ami d'antan lors d'une soirée. Dans ce monde, des puces implantées dans chaque individu dès la naissance permettent de repasser à l'envie tous ses « souvenirs », l'oubli n'existe plus et tout peut être revu. A l'aide de ce procédé, Liam va se convaincre en analysant chaque geste de sa femme, en se les diffusant maintes fois, qu'elle le trompe.

L'épisode montre que l'enregistrement et l'archivage de tous les moments vécus confirme que notre présent peut être sans cesse documenté, rester toujours présent et accessible sans aucun effort ou crainte d'oublier. La mémoire est comprise comme une vidéothèque d'expériences que l'on conserve sous leur forme originale, où l'on appelle l'une d'elles pour la revoir, en découvrir tout ce qu'on aurait pu manquer ce jour-là, ou créer d'autres sentiments. La marge pour réécrire l'original et le modifier est absente. Le passé, constamment revisité par le présent dans son expérience de remémoration, est figé.

L'épisode se concentre sur cet accès devenu obsessionnel, où l'oubli n'a pas de place, la quête des émotions se fait par l'image, la reconnaissance et le surgissement des souvenirs n'est plus gratitude. Le rêve de la maîtrise de la mémoire devient cauchemar et la délivrance par l'oubli semble être la seule voie de sortie possible. La mémoire intégrale du protagoniste le rend malheureux, fou, désespéré, incapable de penser comme Irénée Funès : «J'ai à moi seul plus de souvenirs que n'en peuvent avoir eu tous les hommes depuis que le monde est monde. Mes rêves sont comme votre veille. Ma mémoire, monsieur, est comme un tas d'ordure. (...) Il avait appris sans effort l'anglais, le français, le portugais, le latin. Je soupçonne cependant qu'il n'était pas très capable de penser. Penser c'est oublier des différences, c'est généraliser, abstraire. Dans le monde surchargé de Funès il n'y avait que des détails, presque immédiats » (Borges 1983).

L'incapacité de remémoration et l'hypermnésie y sont dénoncées. Dans la culture numérique l'inversion mémoire et oubli a déjà été constatée : «c'est maintenant par défaut que toute information (sonore, visuelle, textuelle) est 
enregistrée et conservée sous forme digitale - l'oubli nécessitant une action positive d'effacement de données, devenant de ce fait l'exception plutôt que la règle » (Rouvroy 2009, 2).

Pour ce qui est du "contexte de remémoration présent», il est possible d'affirmer qu'il n'est plus la scène mentale d'où peuvent surgir des odeurs, des sons, des mélodies, des rêves, etc. (Stern 2003). Le « contexte» est fermé, les événements ne sont pas ni choisis ni réorganisés dans ce moment présent car ils sont figés, matérialisés par les supports et élaborés par eux.

Sans être aussi obsessionnel dans son rapport au présent des choses passées, il est à noter un autre exemple d'enregistrement : les lunettes noires, orange ou turquoise, de Snapchat appelées Spectacles. Celles-ci permettent d'enregistrer des vidéos de 10 secondes. Une fois filmée, la vidéo est envoyée via Bluetooth ou Wi-Fi puis mise en ligne dans l'application. Snapchat propose ainsi à ses utilisateurs de filmer directement le moment présent, de le capturer et de le partager de façon permanente ou éphémère. Le blog de Snapchat écrit: "Imaginez l'un de vos plus précieux souvenirs. Que diriez-vous de pouvoir remonter le temps et de voir ce souvenir exactement comme vous l'avez vécu? C'est exactement dans cette optique que nous avons conçu nos Spectacles, pour permettre de vous créer facilement plein de souvenirs $»^{1}$.

Ici, la logique du flux et du stockage fonctionne ensemble. D'un côté le flux est renouvelé en continu et met en scène une actualité qui chasse le contenu de la mémoire au fur et à mesure des humeurs exprimées et partagées en ligne, et d'un autre côté, toute activité est stockée et traçable. La distance entre voir et vivre, souvenir et enregistrement, remémorer et créer des souvenirs par l'enregistrement n'est pas en place. Le passé coexiste avec le présent à portée de main ou de click, il n'y a plus à le convoquer, ni à s'inquiéter de l'avoir oublié, il n'y a même plus à le chercher. Davantage encore, il n'y a plus de passé, il est dans un présent figé, sans profondeur, dans la superficie du présent. Il n'est plus nécessaire de faire resurgir des impressions, des émotions, de reconnaître ces traces corporelles : une saveur, une odeur... Car l'enregistrement constant semble donner exactement ce qu'a été vécu, voire plus.

Si précédemment ne savions pas où se tenait caché le souvenir ni comment il survivait, où il fallait aller pour le trouver, dorénavant cette question ne se pose plus puisque nous avons accès à l'endroit, le nuage, où le souvenir est stocké. Il n'est plus important d'analyser les raisons de l'oubli, ses associations, ses refoulés, ses réécritures (Freud 2015, 236-240). Mais le souvenir est-il directement proportionnel à la quantité stockée ? Peut-on dire que plus nous nous sauvegardons, plus nous nous souvenons?

D'une certaine manière on pourrait dire que le présent des choses passées est automatiquement traduit en données digitales. Mais cette traduction ne

${ }^{1}$ Billet du 24 septembre 2016, https://www.snap.com/fr-FR/news/ consulté le 13 avril 2017. 
permet pas une interprétation de la part des individus car ils sont dépossédés de leurs traces, ils sont rendus incapables de partager, de se réapproprier, d'interpréter et de transformer leur mémoire : «Il nous faut en tout cas noter que la " mémoire digitale », à la différence de la « mémoire incarnée» dans les individus ou les collectivités, est une mémoire éminemment mobile, se réorganisant constamment " en temps réel », répondant principalement aux impératifs de pertinence et de vitesse alors que deviennent inopérants les critères de vérité, d'objectivité, de diversité, de critique et de 'profondeur historique' propres à l'évaluation de la mémoire humaine (...) En d'autres termes, la mémoire digitale construit une forme de "savoir » suivant des algorithmes répondant aux besoins bureaucratiques spécifiques du contrôleur des traitements de données ou de son mandant, et qui, pour une large part, échappent à la connaissance et au contrôle des individus auxquels ce savoir se rapporte » (Rouvroy 2009, 12).

La mémoire digitalisée laisse de côté le vécu du temps passé, la perception des temps de passage, mais aussi le flux du présent et la difficulté de la présence. Elle semble s'opposer à l'affirmation qui soutient que tout passe sans rester, comme si jamais aucun moment ne demeurait.

La mémoire digitale semble vouloir donner du temps au temps et une présence sédimentée au présent. Elle croit maintenir ce qui est éprouvé même si on ne peut plus le sentir une fois qu'il est passé. Réempruntant le langage de Saint Augustin, la mémoire digitalisée ressemble à une bande passante qui mesure, compte l'intervalle, mais rate la distentio. L'extension numérique de la mémoire oublie la distentio animi : « tout comme celui qui chante un air connu ou qui écoute un cantique connu, dont l'attente de sons à venir et la mémoire de sons passés fait varier l'affect et se distraire le sentir » (St. Augustin XI, 31, 41, 14).

Selon le vocabulaire de Stiegler, cette mémoire digitale opère une rétention tertiaire. Elle désigne la mémoire sédimentée, objectivée, matérialisée dans des supports inorganiques organisés. Elle articule rétention primaire et rétention secondaire, perception et souvenir. La conscience présente va se constituer à partir des critères de sélection que constituent ces supports.

Pour lui il ne s'agit pas d'opposer temporalité authentique et intra-temporalité technique, mais de voir comment la notion de rétention tertiaire ouvre de nouvelles possibilités de conservation de la mémoire. Il ne s'agit pas non plus d'opposition entre intériorité et extériorité mais d'une relation qui constitue ses termes qui n'existent pas hors de la relation. L'expérience temporelle des individus se sédimente et devient partageable, transmissible. La question est de savoir de quelle manière ils vont recevoir et interpréter ces expériences, comment ils vont les transformer à travers leurs pratiques et quelles possibilités ouvre un avenir qui ne peut être calculé.

Stiegler montre aussi que l'accélération des innovations technologiques, exploitées par le capitalisme computationnel et le marketing stratégique, 
court-circuite aujourd'hui les temps de réflexion et de délibération. Le courtcircuit temporel fait en sorte que les temps vécus ne soient plus ceux de la mémoire, de l'attente ni de l'attention.

On trouve une dénonciation très claire de cette accélération chez Harmut Rosa. Les expériences classiques du temps vécu, où l'intervalle long/bref est l'intervalle habituel, a été remplacé par un intervalle bref/bref. Ce qui veut dire pour lui que le temps vécu présent passe très vite et qu'il rétrécit dans la mémoire. D'ailleurs n'est-il pas vrai que nous peinons souvent à nous souvenir d'avoir été quelque part, d'avoir fait quelque chose, d'avoir vu quelqu'un?

Le langage augustinien dirait que l'attention ne fait plus transiter ce qui était futur pour devenir passé, c'est-à-dire qu'elle ne peut plus se situer dans le mouvement du multiple ni assurer son unité (distentio). La coexistence dans le présent de ces modalités temporelles n'est plus assurée ${ }^{2}$.

L'attente, l'attention et la mémoire ne sont pas en interaction : "Nous échouons à faire du temps de nos expériences "notre temps» : les épisodes d'expérience, et le temps qui leur est alloué, restent pour nous étrangers. Un manque d'appropriation de nos propres expériences, cependant, ne peut que mener à des formes plus- plutôt que moins- sévères d'auto-aliénation » (Rosa 2012, 132).

Pour notre auteur, la dissolution du présent rend le sujet étranger et dessaisi du passé. Pas de pause, pas de silence, eux-mêmes sont au service de l'accélération. Cette aliénation provoque un oubli des jours, des êtres et des actions et une étrangeté du sujet à son propre temps, une absence de sa présence. Ceci est pour Rosa le résultat d'une aliénation « dont le cœur est une distorsion temporelle poussée de la relation moi-monde » (Rosa 2012, 138). Portés par la flexibilité temporelle, les moments n'arrivent même plus à être une succession repérable et les actions qui les accompagnent se présentent sans lien significatif les unes avec les autres. Combien de fins de journées vont se clore sans le souvenir d'elles-mêmes, sans mémoire, et pourtant, n'avonsnous pas cessé de courir derrière le temps et de remplir toute sorte de calendriers?

La fonction d'orientation et de stabilité du caractère unilatéral du temps, grâce aux calendriers et aux aiguilles du cadran, se noie dans la fluidité propre au devenir pour laisser place au règne de «l'amnésie du quotidien ». Le fil de la journée est coupé, une infinité de trames se tissent et nous les parcourons sans nous retourner. Le fil d'une seule heure s'efface, et une succession de secondes flexibles s'écoule sans notre présence. Le présent des choses passées et présentes devient oublié, méconnu et muet.

2 Saint Augustin, Les Confessions, XI, 28, 38: « et les forces vives de mon activité sont distendues vers la mémoire à cause de ce que j'ai dit et vers l'attente à cause de ce que je vais dire. Mon attention est pourtant là, présente ; et c'est par elle que transite ce qui était futur pour devenir passé ». 


\subsection{Le présent otage de lui-même}

Selon Harmut Rosa, le constat de l'accélération va de pair avec celui de "l'immobilité fulgurante» (Virilio 2002). L'orientation vers l'avenir est suspendue. L'élan du coureur du praesens est immobile, penché en avant, le mouvement auquel il est contraint par l'injonction sociale d'accélération lui fait perdre toute possibilité d'impulsion. Il est figé dans une roue sans avoir recours à la mesure ni aux rythmes. L'accélération qui nous laisse à court de temps, met à court de souffle et coupe l'élan pour ce qui est à l'avant, au-delà.

Dans cette distorsion temporelle où le futur est " coagulé » apparaît une sorte d'espace où toutes les simultanéités et multi-temporalités ont de la place. Le vécu devient alors adhérence à la fluidité. Quant à la simultanéité elle détend les contraintes d'une mesure et d'un rythme. Ce temps informe, presque inerte devient en quelque sorte un éternel prêt à être consommé : " C'est la vitesse, et non la durée qui compte. Avec la bonne vitesse, on peut consommer toute l'éternité à l'intérieur du présent continu de la vie terrestre (...) L'astuce est de comprimer l'éternité de façon qu'elle entre toute entière, dans le temps de la vie individuelle » (Bauman 2006,17).

L'adhésion à la fluidité célèbre l'irréversible qui favorise la métamorphose et qui n'a nul besoin de l'oubli ou de la mémoire pour continuer à nous emporter d'instant en instant : "l'irréversible nous entraîne de nouveauté en nouveauté, chacune innovant sur la précédente, sans que nous ayons le temps pour méditer, ni d'approfondir le sens de chaque expérience; les impressions, emportées sans trêve ni pause par la continuité de la futurition, se refoulent l'une l'autre dans le néant, et glissent sur nous sans laisser la moindre trace, du moins l'impression, vite effacée, reste-t-elle superficielle et fugace » (Jankélevitch 1980, 93).

Ainsi l'expérience du présent, glisse sans laisser de traces et met en relief l'impuissance du sujet à arrêter son écoulement. Les risques d'une telle « célébration » sont multiples : l'oubli du passé, l'aliénation du temps vécu, le manque de lien entre les instants, la désagrégation et la dissolution dans un présentisme. Ces risques se sont réalisés et nous y assistons. «Aucun espoir pour l'avenir », "Plus de temps pour rien » voilà des formules bien souvent entendues ou prononcées! L'avenir y apparaît suspendu, l'histoire sans histoires, et le présent en accélération constante. Rien d'étonnant à ce qu'il y ait une plainte constante sur le manque de temps, rien d'étonnant non plus à ce qu'apparaissent des «pathologies temporelles » telles que la précarisation des projets à long ou moyen terme, qu'ils soient individuels ou sociaux, ou telles que l'impossibilité et l'impuissance à faire advenir ou à attendre ce qui survient.

Le futur devient prêt à être toujours un présent flexible, adaptable, prévisible, anticipatif. Le futur et le présent des choses passées et des choses présentes n'entretiennent plus de relations, ils sont désarticulés et leur métamorphose crée un "présent monstre», presque rien et tout (Hartog 2012, 270). Selon 
Hartog, «le futurisme s'est abîmé sous l'horizon et le présentisme l'a remplacé. Le présent est devenu l'horizon. Sans futur et sans passé, il génère $\mathrm{au}$ jour le jour, le passé et le futur dont il a, jour après jour, besoin et valorise l'immédiat » (Hartog 2012, 157).

\subsection{Le présent des choses à venir est programmé}

C'est à partir de Bernard Stiegler et grâce à sa catégorie de disruption que la disparition de l'attente est dénoncée. C'est-à-dire la dissolution du présent des choses à venir qui est l'attente en faveur de l'anticipation. Cette catégorie de disruption est définie comme l'impasse de l'ère anthropocène ${ }^{3}$ qui concrétise et accomplit le nihilisme4.

Par la disruption, les individus et les réseaux sociaux se trouvent «désindividués par le fait même que leurs propres données, qui constituent aussi ce que l'on appelle (dans le langage de la phénoménologie husserlienne du temps) de rétentions, permettent de les déposséder de leurs propres protentions - c'est-à-dire de leurs propres désirs, attentes, volitions, volonté, etc. » (Stiegler 2016, 23). Autrement dit, les individus ne possèdent plus leur passé ni la dynamique de leurs attentes dans le temps présent. Le passé inscrit dans les données incite à répéter des schémas à n'importe quelle échelle et dans n'importe quelle condition. Les degrés de liberté dans le présent sont bien réduits.

La fin de tout rêve, de toute attente, de tout désir est actée tout au long du livre. L'automatisation intégrale et généralisée de la mémoire et de l'attente conduit à affirmer que «nous sommes jetés dans et par l'époque de l'absence d'époque ». L'absence de présent passé et de présent futur empêche tant au niveau individuel que collectif la transmission et la création de nouvelles formes de culture. Le renouvellement n'est pas possible puisqu'il n'y a plus d'horizon d'attente commun aux individus. Une faiblesse, une apathie, une perte de la raison de vivre s'est installée. La promesse, l'au-delà ne font plus partie de l'horizon d'attente, personne ne veut l'avenir. Les attentes psychiques sont remplacées par des attentes automatiques.

Stiegler dénonce la perte de la raison, la société automatique, la destruction des liens sociaux, la prise du contrôle de l'imagination, la confusion entre les choix libres et la production des clicks, la destruction de tout pouvoir symbolique, de tout processus d'identification intergénérationnelle, filiale,

3 « La disruption est ce qui, advenant dans l'ère géologique de l'Anthropocène, et comme son impasse même, empêche structurellement la formation de protentions collectives porteuses d'un avenir chargé de puissance nouvelle au moment où la possibilité imminente d'une ubris si l'on peut dire démesurément et définitivement fatale étreint et étrangle toute projection dans l'immensité de l'improbable, et, ce faisant, rend fou - fou de tristesse, fou de douleur, fou de rage » (Stiegler 2016, 42-43).

4 «L'anéantissement algorithmique de la promesse est une annihilation, ce qui nous reconduit au fait que nous vivons l'époque de l'accomplissement du nihilisme, sinon du nihilisme tout à fait accompli : nous vivons la phase dans laquelle la catastrophé nihiliste est en train de se déployer » (Stiegler 2015, 72). 
individuelle, l'élimination de la faculté de rêver, la dissolution algorithmique du social.

Il ouvre deux possibilités de sortie: soit on sort de la disruption comme absence d'époque soit le genre humain finira :

Qu'un renouveau de la noétique soit possible ne signifie pas qu'il est probable: le possible est souvent si improbable qu'il se présente avant tout comme l'impossible. Cet impossible peut et doit se réaliser et comme rêve de l'improbable: faute d'y parvenir, la non-inhumanité est condamnée à disparaître, soit dans l'oligarchie dominant une termitière nano-biotechnologique (...) soit, bien plus probablement dans une augmentation irréversible du taux d'entropie tel que la vie ellemême se trouvera menacée, du moins dans ses formes évoluées (Stiegler 2016, 240).

$\mathrm{Ni}$ pessimiste ni optimiste, il affirme que le pharmakon numérique est aussi porteur d'une nouvelle néguentropie qui bifurquerait au-delà du chaos disruptif (Stiegler 2016, 76-77). Cela appelle à une transformation radicale de l'innovation et requiert courage et volonté de lutter, d'analyser, de critiquer, de laisser des traces, d'assumer "l'indétermination radicale du futur comme avenir irréductible au devenir » (Stiegler 2016, 107) et de tout faire pour en inverser le cours.

La ligne à suivre consiste selon lui à se projeter par-delà l'Anthropocène, dans le néguanthropocène. Cette bifurcation passe par le rêve de la raison qui «se mesure au chaos » :

La faculté de rêver est à l'origine de la noèse parce que celle-ci est de part en part et originellement néguanthropologique : en tant que source de toutes les formes de savoir (...) la noèse est ce qui ouvre des possibilités de bifurcations inscrites dans le réel, mais qui ne sont accessibles que comme et par la déréalisation épokhale de ce réel (Stiegler 2016, 287).

L'attente, le désir semble extenué, mais l'impossible, même improbable devient le rêve. Cet improbable n'a pas, selon notre auteur, à se constituer dans une foi en Dieu, ni dans l'attente d'une divinité qui pourrait encore nous sauver, mais cet improbable peut donner lieu à créer du sens dans l'immonde et reconnaître dans l'enfer qui est devenu l'époque sans époque ce qui n'est pas enfer, lui faire place : "Comment une conversion pourrait-elle se produire à partir de l'absence d'époque? En faisant des mondes dans l'immonde, en redonnant lieu, en faisant proliférer l'avoir lieu en mille lieux où faire la différence. C'est ce que nous disent René Char et Italo Calvino, faisant naître dans les lieux de l'urbanité les miraculeuses relations d'admiration mutuelle où recule l'enfer devant ce qui au milieu de l'enfer, n'est pas l'enfer, et le faisant durer, et lui faisant place » (Stiegler 2016, 440-444). 
Le présent des choses à venir c'est l'attente mais celle-ci n'a plus de forme, les algorithmes d'anticipation, de prédiction, de recommandation éliminent tant l'impatience d'espérer comme le surgissement du désir dans les plis du présent. C'est pourquoi Stiegler appelle à faire en sorte que la question de : « la renoétisation par la reconstitution du désir - dont la raison est d'abord le motif, c'est-à-dire le moteur, et, en cela, la dynamique protentionnelle dans son ensemble (...) doit être affrontée face à l'état d'urgence qu'est la disruption » (Stiegler 2016, 427). Affronter l'exténuation du désir demande une conversion dans les façons de vivre, d'appréhender le temps présent : «la conversion à venir est celle du devenir en avenir » (Stiegler 2016, 429-430) et de croire à la possibilité d'un miracle à tout moment.

\section{Il est temps de créer dans le présent}

Quand on énonce "qu'il est temps » nous énonçons l'urgence de reconnaître les brèches dans les expériences temporelles actuelles par lesquelles la création avec elles soit possible. Puisque comme il est écrit dans le fragment 18 d'Héraclite: Qui pour lui-même n'espère pas l'inespéré, il ne trouvera pas : c'est introuvable autant qu'inaccessible.

Le présent sous-tend le mouvement en avant, il bouleverse ce qui est attendu et son issue est imprévisible, l'incertitude peut être totale. La réduction de l'instant au flux irréversible, la prédictibilité du futur, le traitement algorithmique des traces du passé, ont leur revers: latence, désir, reconnaissance, promesse, rêve, impossible... Ce présent est une histoire qui a une infinité de narrations et d'interprétations.

\subsection{La brèche dans l'instant}

La modification du temps présent ne semble faire aucun cas de l'émergence, de la créativité, de l'apparition, de l'altérité contenue dans le présent des choses présentes. Ce qui apparaît devient apparence statique. L'instant qui est le propre du présent du devenir s'épuise et nous épuise. Nous ne voyons plus l'être latent qu'il porte en lui. C'est comme si ces diktats avaient préféré homogénéiser et pétrifier le présent du devenir.

Ces diktats préfèrent ignorer que la succession des instants déroule une palette infinie de nuances, de tonalités différentes, de nouveautés constantes. Ils soutiennent l'irréversible dans son côté d'épuisement, de déchet, de succession sans signification, d'angoisse, de stress, et ignorent tout ce qui est de l'ordre de la promesse dans l'altération, de la maturation dans le changement, de la nouveauté dans le surgissement. Ils donnent tout leur appui à la vitesse, à l'apparaître, à l'homogène oubliant qu'il est possible de faire confiance au présent du devenir.

L'oubli des jours fait en sorte que leur différence s'éclipse dans une ligne identique. Dans cet « il y a en temps réel» tous les instants deviennent traces 
sans contexte: "Comme le rappelle Bruno Bachimont, "la manipulation algorithmique ou calculatoire [du signe informatique] n'est possible que si elle porte sur des éléments discrets dépourvus de signification". De fait, au niveau d'un clic ou d'une fraction de log, la trace ne signifie rien. Mais, précisément parce qu'elle n'est pas sémantique, elle est détachable de son contexte et susceptible d'être croisée avec d'autres "unités isolables, agençables et calculables" » (Merzeau 2012). L'arôme inimitable de l'instant, son charme, et les occasions qu'il contient sont balayés par la traçabilité qui se soustrait au caractère polymorphe des instants.

Ce perpetuum mobile ne sait pas grand-chose du futurum esse. Il n'a ni la patience ni l'espérance de ce qui est en train d'advenir, en instance d'advenir. Il singe le mouvement mais a perdu tout rythme. Il imite les dynamismes mais il ne fait qu'échouer sur l'immutabilité.

Les expériences temporelles dans l'horizon qui souligne exclusivement un des côtés du caractère irréversible du devenir ont conduit à des dénonciations diverses telles que le dessaisissement du temps vécu et son étrangeté, l'amnésie du quotidien, l'effacement du passé et du futur, le règne d'un présent monstre et caricatural, la désindividuation...

Ces dénonciations veulent attirer l'attention sur les diktats de suppression des temps et l'impuissance de ces instants irréversibles à couver le futur. Elles veulent mettre en évidence des aliénations personnelles et sociales, décrire de nouveaux régimes d'historicité, dénoncer la culture du déchet, la désorientation, les vies égarées. Elles se pensent comme voies prometteuses pour ouvrir des espaces de critique et de pensée pour des vies non aliénées.

Or l'autre côté du flux est à récupérer : il n'est pas qu'un mouvement perpétuel et fade qui transforme en déchet chaque instant. Ce flux toujours naissant héberge dans chaque instant une heure propice, un kairos.

La créativité consisterait à étendre et déplier ce qui est roulé sur soi-même dans l'instant. Voilà qui rendrait vertigineuse la décision d'advenir, hasardeuse la résolution de reconstruire ses expériences temporelles. Ce serait continuellement faire acte de mémoire, reconnaître des lieux et cultiver un amour des temps.

Je dis bien « cultiver », car penser le devenir qui se déploie sans cesse n'est pas sans évoquer les idées de semence, d'éclosion, d'épanouissement, de fleurissement et même de culture et d'entretien, peut être aussi celles de maturation qui conduit au déclin, à l'affaiblissement, au vieillissement, à la caducité... Fleurir/faner... s'épanouir et s'étioler jusqu'à sécher... Il y a dans ces mots une évocation analogique qui peut ouvrir à la compréhension de ce devenir. Comme modification continuée, sous un apparent modelage, le temps se défait sans cesse, se forme, se déforme, se reforme et se transforme, il 
modèle et métamorphose chaque être. Ne serait-il pas possible de regarder chaque instant comme une révélation continuée ?5

\subsection{Quelques fissures en vue du futur}

"Nous avons une mémoire, mais nous sommes le souvenir » affirme Lou Andreas-Salomé, de sorte que le souvenir serait un accomplissement "poétique ». Chacun d'entre nous a une partie réservée de poésie qui résulte d'un passé où la distance s'est établie et d'un présent où s'élance l'advenir. Voilà l'écart : le souvenir et son refoulement restent matière de création.

L'enregistrement des données n'a pas pris en compte les refoulements, l'extension de nos souvenirs, l'élargissement de notre présent des choses passées. Si l'art pour Freud est un remède sans égal pour le refoulement, la créativité peut prendre place dans cet espace et délivrer de la rigidité, de l'immobilité et des confusions que nous avons dénoncées. Sans être influencé par notre mémoire numérique à notre sujet, l'espace est déjà sauvé, la mémoire continue en secret créatrice de souvenirs et de rêves.

Le rêve a été comparé à l'œuvre d'art primitive, la création nocturne donne au chaos la force d'une forme. Dans le rêve éveillé le passage à l'activité se prépare si le désir s'écarte de la domination de ses propres idées et de ses mémoires numériques.

Une autre fissure est celle de rêver l'impossible, de rêver la réalité comme l'annonçait Stiegler. Elle est comme un élément autre à l'intérieur de l'obsession mémorielle, comme une sorte de tension quichottesque qui se heurterait à la disruption et qui ferait exploser toutes les expériences temporelles ainsi que celles de l'espace.

Une dernière entaille encore est celle de l'art de l'oubli. Il ne s'agit pas là d'un droit à l'oubli à la faveur de la liquidité, ou d'un oubli conçu comme un disfonctionnement de la mémoire. A la fin de l'épisode de la série citée précédemment, le personnage s'enlève la puce et rentre dans l'oubli comme libération. Entre la menace d'un oubli définitif et d'une mémoire totale, entre l'impression du présent dans nos vies et la survivance des affections, la reconnaissance a une place unique : « elle est l'acte amnésique par excellence » (Ricœur 2000, 557). Bref, en reprenant les mots de Heidegger : « De même que l'attente n'est possible que sur la base d'un s'attendre, de même le souvenir n'est possible que sur la base d'un oublier et non pas l'envers » (Ricœur 2000, 573).

Attendre c'est désirer, et pour Stiegler l'objet du désir reste toujours Dieu : «C'est l'objet de tous les désirs. C'est-à-dire le désir de tous les désirs. Et c'est en conséquence l'objet de toutes les attentions. Ce que le nom Dieu a nommé, c'est l'objet de toutes les attentions : l'attente absolue d'un avenir absolu qui contient tous les désirs. Qu'on veuille lui donner le nom de Dieu ou pas m'est

\footnotetext{
${ }^{5}$ Des variations réflexives à partir de l'œuvre de Vl. Jankélévitch.
} 
absolument égal » (Stiegler 2016, 463). Pour ma part qu'on veuille lui donner le nom de Dieu ne m'est en rien égal. Il est par contre absolument nécessaire.

\section{Littérature}

Bauman, Z. 2006. La vie liquide. Paris: Pluriel.

Benveniste, E. 1966. Le système sublogique des prépositions en latin. In Id. Problèmes de linguistique générale I. Paris: Gallimard.

Borges, J. L. 1983. « Funès ou la mémoire ». In Id. Fictions, Paris: Gallimard.

Freud, S. 2015. «Sur le mécanisme psychique de l’oubli (1898) ». In R. Perron, S. Misonnier (dir.), Freud. Paris: L'Herne, 236-240.

Hartog, Fr. 2012. Régimes d'historicité. Présentisme et expérience du temps. Paris: Seuil.

Jankélévitch, Vl. 1980. Le je-ne-sais-quoi et le Presque-rien, vol. II. Paris: Seuil.

Merzeau, L. 2012. "Faire mémoire des traces numériques". E-dossiers de l'audiovisuel, Sciences humaines et sociales et patrimoine numérique. [En ligne] http://www.ina-expert.com/e-dossier-de-l-audiovisuelsciences-humaines-et-sociales-et-patrimoine-numerique/fairememoire-des-traces-numeriques.html.

Ricœur, P. 2000. La mémoire, l'histoire, l'oubli. Paris: Seuil.

Rosa, H. 2012. Aliénation et accélération. Vers une théorie critique de la modernité tardive. Paris: La Découverte.

Rouvroy, A. 2009. "Réinventer l'art d'oublier et de se faire oublier dans la société de l'information? ». In S. Lacour (dir.), La Sécurité de l'individu numérisé. Réflexions prospectives et internationales. Paris: L'Harmattan.

Stern, D. N. 2003. Le moment présent en psychothérapie. Un monde dans un grain de sable. Paris: Odile Jacob.

Stiegler, S. 2015. La société automatique I. L'avenir du travail. Paris: Fayard.

Stiegler, S. 2016. Dans la disruption. Comment ne pas devenir fou? Paris: Les Liens qui libèrent.

Virilio, P. 2002. L'inertie polaire. Paris: Christian Bourgois. 
Gemma Serrano

(Faculté de Théologie Notre-Dame, Collège des Bernardins, Parisgemma.serrano@collegedesbernardins.fr)

\title{
Le présent altéré
}

\begin{abstract}
English). Our working hypothesis is that the digital culture is remodeling a temporal affect of existence. We attempt to describe how our experience of present time is altered by the increase of digital memory, together with the progressive disappearance of " memory recall » and expectation. This altered presence opens up loopholes which could be called dreams, desire, promise, latency or God. Thus anticipatory algorithm and data translation of present actions of the subject are not a definitive tragedy of our time.
\end{abstract}

Abstract (François). Notre hypothèse de travail est que « le numérique » est en train d'instaurer un nouvel affect temporel de l'existence. Notre propos consistera à décrire l'altération de l'expérience du temps présent par l'accroissement de la "mémoire digitale », la progressive disparition de la remémoration et la disparition de l'attente. Ce présent altéré ouvre des brèches nommées rêve, désir, promesse, latence, Dieu. L'anticipation algorithmique et la traduction en données des actions présentes du sujet n'est pas définitivement une tragédie des temps.

Keywords: digital culture, digital memory, look-ahead algorithm, memory recall, expectation, present, creativity

Mots-clés: digital numérique, mémoire digitale, données, anticipation, créativité, remémoration, attente, présent

Ethics in Progress (ISSN 2084-9257). Vol. 8 (2017). No. 1, Art. \#5, pp. 75-88.

Creative Commons BY-SA 3.0

Doi: 10.14746/eip.2017.1.5 